\title{
Silica twisted and helical nanoribbons as chiral inducers for peroxophosphotungstate anions.
}

\author{
Mariam Attoui, ${ }^{[\mathrm{a}, \mathrm{b}]}$ Emilie Pouget,${ }^{[\mathrm{a}]}$ Reiko Oda ${ }^{[\mathrm{a}]}$ David Talaga, ${ }^{[\mathrm{b}]}$ Thierry Buffeteau, ${ }^{* \mathrm{~b}]}$ and Sylvain \\ Nlate*[a] \\ ${ }^{a}$ UMR 5248 CBMN, CNRS-Université de Bordeaux-ENITAB, Institut Européen de Chimie et Biologie, 2 rue \\ Robert Escarpit, F-33607 Pessac, France. Fax: (+33) 5-4000-2215, E-mail: s.nlate@iecb.u-bordeaux.fr \\ ${ }^{b}$ Institut des Sciences Moléculaires (ISM), CNRS, UMR 5255, Université de Bordeaux, 351 Cours de la \\ Libération, 33405 Talence.
}

\begin{abstract}
As a part of our ongoing research towards the investigation of chiral polyoxometalate-based nanomaterials (NANOPOMs), new chiral POM-based silica twisted and helical ribbons were synthesized by the treatment of 3-aminopropyl triethoxysilane (APTES) functionalized left- and right-handed silica twisted and helical nanoribbons with phosphotungstic acid $\mathrm{H}_{3} \mathrm{PW}_{12} \mathrm{O}_{40}$ and excess of $\mathrm{H}_{2} \mathrm{O}_{2}$. The morphology and structural composition of the resulting NANOPOM hybrids as well as the presence of POM species were confirmed by various physicochemical techniques such as solid ${ }^{31} \mathrm{P}-\mathrm{NMR}$, UV-Vis, Raman, TEM, HR-TEM, and EDX. Solid ${ }^{31} \mathrm{P}$ NMR studies indicate that the structures of these NANOPOM hybrids contain various phosphotungstate anions, with among them, the catalytically active Venturello peroxophosphotungstate trianion $\left[\mathrm{PO}_{4}\left\{\mathrm{WO}\left(\mathrm{O}_{2}\right)_{2}\right\}_{4}\right]^{3-}$. Interestingly, the optical activity of these twisted and helical NANOPOM hybrids has been confirmed by circular dichroism (CD) spectroscopy, highlighting the chirality induction from chiral nanoribbons to achiral POM clusters. The catalytic performances of these NANOPOM hybrids were evaluated in the heterogeneous oxidation of methyl phenyl sulfide as a model reaction, indicating NANOPOM hybrids built from twisted ribbons as the most efficient catalysts.
\end{abstract}

Keywords: chiral silica nanostructures; peroxophosphotungstate; twisted and helical nanoribbons; circular dichroism ; chirality induction 


\section{Introduction}

Polyoxometalates (POMs) [1] are an important class of inorganic compounds with highly interesting properties that render them attractive candidates for potential applications in a variety of fields such as catalysis, bio-medicine, optics and nanomaterials [2]. Among their diverse functional properties, the chirality of POMs attracts much wider interest, due to the enormous versatility that POMs offer in structural templating, asymmetric catalysis and optics. In this context, assembly of chiral polyoxometalates (POMs)-based materials combines the functionality from both POMs and chiral materials, which can results in improved POM properties [3]. Although POMs exhibit good activity in homogeneous processes, the main disadvantage for practical application lies in their low surface areas and the difficulty to separate them from the reaction mixture. To overcome these drawbacks, the coupling of POMs with soluble supports such as dendrimers [4], or their immobilization onto solid supports [5] to create hybrid materials have attracted particular research interest due to their easy recycling. Among chiral POMs-based materials reported so far, helical selfassembled supramolecular architectures have received more attention in the last few years because of their attractive structural feature and interesting properties. Many approaches are proposed for the preparation of POM-based helical materials generally obtained by chirality induction through attachment of chiral unit as a chirality transfer agent onto the POM [6], or by spontaneous symmetry breaking of POM upon crystallization in the absence of any chiral source, the latter being the most used strategy for the preparation of chiral POM-based materials [7]. The ability to design and fabricate POM-based helical nanostructures with controlled size, in which POM units are organized onto the helical matrix following the helical pitch remains an important challenge in this area of research. Such organization of POMs is expected to bring new optical properties to the whole NANOPOM hybrid material. Thus, a well-defined helicity and strong interactions between POM units and the helical matrix are required for the expression of chiroptical properties of POMs within such POMbased materials. Whereas helically organized Gold Nanoparticles (GNPs) are well developed and show interesting chiroptical properties [8,9], nanomaterials in which POM clusters have been organized on helical structures are rare. Recently, the achiral Keggin anion $\left[\mathrm{PW}_{12} \mathrm{O}_{40}\right]^{3-}$ has been grafted onto silica nanohelices by electrostatic and direct adsorption interactions, and showed the induced chirality from the chiral silica nanohelices[10]. An area of particular interest to us is to investigate how a soluble or solid support may alter the properties of the incorporated POMs. In this context, we have shown few years ago that the incorporation of 
the Venturello peroxophosphotungstate trianion $\left[\mathrm{PO}_{4}\left\{\mathrm{WO}\left(\mathrm{O}_{2}\right)_{2}\right\}_{4}\right]^{3-}[11]$ into various dendritic cations [4c-h] can resulted in the modification of the POM properties. We found that the nature of the dendritic cation is closely related to the properties (catalytic efficiency, stability, solubility, recyclability and optical activity) of the POM clusters. In the case of chiral dendritic structures, the chirality is manifested in the whole DENDRIPOM hybrid, highlighting the transfer of chirality to POM clusters. These studies indicated that the properties of POM anions can be modulated by varying the nature of the counteranion. Whereas various POM clusters have been grafted onto solid supports, POM-based materials in which peroxophosphometalate anions have been immobilized onto inorganic solid materials are under represented. Of those reported so far, to the best of our knowledge, no report describes the assembly of peroxophosphometalate with helical structures, and the characteriation of the resulting helical NANOPOM materials as well as their chiroptical properties investigation. In this context, as a continuation of our studies on chiral peroxophosphotungstate based materials, we have investigated the immobilization of peroxophosphotungstate trianion $\left[\mathrm{PO}_{4}\left(\mathrm{WO}\left(\mathrm{O}_{2}\right)_{2}\right)_{4}\right]^{3-}$ onto two chiral silica supports, namely left- and right-handed twisted and helical nanoribbons, and studied the chiroptical properties of the resulting NANOPOM hybrids. Nanostructures obtained by supramolecular organization of organic molecules are known to be very sensitive to chemical, mechanical and thermal perturbation [12]. Thus, much effort has been made to develop more stable inorganic chiral nanostructures [9a,13]. Our approach for the preparation of these chiral peroxophosphotungstate-containing materials is based on the treatment of aminefunctionalized silica twisted and helical nanoribbons with phosphotungstic acid $\mathrm{H}_{3} \mathrm{PW}_{12} \mathrm{O}_{40}$ and excess of $\mathrm{H}_{2} \mathrm{O}_{2}$.

Herein, we described our attempt to prepare NANOPOM hybrids based on the combination of the Venturello peroxophosphotungstate trianion and silica twisted and helical nanoribbons respectively.These NANOPOM hybrids display chiroptical properties, as a result of the chirality transfer from silica nanoribbons to the POM cluster. The morphology and the presence of POM anions within NANOPOM hybrids, as well as the interactions between POMs and silica nanoribbons were investigated by solid ${ }^{31} \mathrm{P}-\mathrm{NMR}$, UV-Vis, Raman, TEM, HR-TEM, and EDX. Whereas solid ${ }^{31} \mathrm{P}$ NMR indicates the presence of the Venturello peroxophosphotungstate $\left[\mathrm{PO}_{4}\left\{\mathrm{WO}\left(\mathrm{O}_{2}\right)_{2}\right\}_{4}\right]^{3-}$ within the structures of NANOPOMs, the presence of signals in $\mathrm{CD}$ spectroscopy highlights their optical activities, as a result of the chirality induction from chiral nanoribbons to achiral POM clusters. These NANOPOM hybrids were evaluated as recoverable catalysts in the oxidation of methyl phenyl sulfide with 
$\mathrm{H}_{2} \mathrm{O}_{2}$, as a model reaction. The effects of the nature of the chiral support (twisted ribbons versus helical ribbons) on the properties of POMs are emphasized.

\section{Experimental}

\subsection{Materials}

Reagent-grade solvents and all chemicals were used as received. Organic oxidation products were identified by correlation to authentic samples.

2.2. Preparation of peroxophosphotungstate-based silica twisted (L)-8 and (D)-8, as well as helical (L)-9 and (D)-9 nanoribbons

2.2.1. Synthesis of gemini tartrate $(L)-1$ and $(D)-1$, organic twisted ribbons $(L)-2$ and $(D)-2$, as well as organic helical ribbons (L)-3 and (D)-3

The 16-2-16 Gemini amphiphile with tartrate counterions $(L)-\mathbf{1}$ and $(D)-\mathbf{1}$ were prepared according to a previously reported procedure [14]. To obtain the organic gel at the concentration of $1 \mathrm{mM}, 3.6 \mathrm{mg}$ of 16-2-16 L- or $D$-tartrate powder were dissolved into $5 \mathrm{ml}$ of ultra pure water. The solution is heated up to $60^{\circ} \mathrm{C}$ (above the Krafft temperature which is $43^{\circ} \mathrm{C}$ for 16-2-16 tartrate) for $20 \mathrm{~min}$ to obtain a complete dissolution and incubated at $20^{\circ} \mathrm{C}$. Twisted ribbons $(L)-\mathbf{2}$ and $(D)-\mathbf{2}$ were observed after $2 \mathrm{~h}$, whereas helical ribbons $(L)-\mathbf{3}$ and (D)-3 were observed after 2 days. The morphology of self-assembly was monitored by Transmission Electron Microscopy (TEM), after a negative staining with 3 wt\% uranyl acetate.

2.2.2. Preparation of silica twisted nanoribbons (L)-4 and (D)-4 as well as helical nanoribbons (L)-5 and (D)-5.

After the preparation of organic gels, the later were used as template to prepare silica nanofibers through a sol-gel transcription method. In a typical procedure, $500 \mu \mathrm{L}$ of tetraethoxysilane (TEOS) were added to the $10 \mathrm{~mL}$ of $0.1 \mathrm{mM}$ aqueous solution of $(L)$-tartaric acid ( $\mathrm{pH} 3.8$ ) and prehydrolyzed at $20^{\circ} \mathrm{C}$ by stirring on the roller-mixer for $7 \mathrm{~h}$. In parallel, a solution of $1 \mathrm{mM}$ 16-2-16 gemini surfactant with $(L)$-tartrate counterion is aged between $2 \mathrm{~h}$ and 2 days for the formation of twisted nanoribbons $(L)-\mathbf{4}$ and $(D)-\mathbf{4}$ as well as helical nanoribbons $(L)-5$ and $(D)-5$, respectively. Equal volume of prehydrolyzed TEOS $(5 \mathrm{~mL})$ in $0.1 \mathrm{mM}$ aqueous solution of $(L)$-tartaric acid and organic gel $(5 \mathrm{~mL})$ are mixed and stirred at $20^{\circ} \mathrm{C}$ with a roller-mixer overnight. Once the transcription is completed, the mixture thoroughly is washed five times with isopropanol in order to eliminate the excess of TEOS 
and the organic template. Finally, the suspension of silica fibers is stored in pure ethanol. The morphology of the later was checked by TEM. Basically, we obtained $5 \mathrm{mg}$ of silica nanoribbons from $1 \mathrm{mM}$ of organic gel.

\subsubsection{Preparation of amine functionalized silica twisted nanoribbons $(L)-6$ and $(D)-6$ as well} as helical nanoribbons $(L)-7$ and (D)-7.

For electrostatic grafting of POM clusters at the surface of silica nanohelices, the silica nanohelices were functionalized with (3-aminopropyl)triethoxysilane (APTES) [9b]. Generally, $20 \mu \mathrm{mol}$ of APTES in absolute ethanol was added per $1 \mathrm{mg}$ of silica nanohelices in ethanol. The reaction mixture was sonicated in ultrasonic bath for $5 \mathrm{~min}$ and heated for $15 \mathrm{~h}$ at $70^{\circ} \mathrm{C}$. After completion, the modified silica nanostructure was washed 3 times with absolute ethanol and then 2 times with water. This procedure can be repeated once to improve the coverage of silica surface with amine groups. The quantity of amine groups at the surface of silica nanohelice was estimated as reported in ref. [9a].

\subsubsection{Silica NANOPOM twisted ribbons $(L)-8$ and (D)-8 as well as helical nanoribbons $(L)-9$} and (D)-9 built by electrostatic coupling of POM clusters with amine functionalized silica nanoribbons.

For the preparation of peroxophosphotungstate anion, phosphotungstic acid $\mathrm{H}_{3} \mathrm{PW}_{12} \mathrm{O}_{40}$ $\left(10^{-5} \mathrm{~mol}, 28.8 \mathrm{mg}\right)$ was dissolved in water $(0.1 \mathrm{~mL})$, and 587 equiv of hydrogen peroxide $35 \%(0.5 \mathrm{~mL}, 5.87 \mathrm{mmol})$ was added to the mixture. The latter was stirred for $30 \mathrm{~min}$ at room temperature, and this mixture was added to a water suspension of $5 \mathrm{mg}$ of amine functionalized silica twisted nanoribbons $(L)-6$ and $(D)-6$ or helical nanoribbons $(L)-7$ and (D)-7. The mixture was stirred at $20^{\circ} \mathrm{C}$ with a roller-mixer for $4 \mathrm{~h}$. The corresponding silica NANOPOM twisted ribbons $(L)-\mathbf{8}$ and $(D)-\mathbf{8}$ as well as helical nanoribbons $(L)-\mathbf{9}$ and $(D)-\mathbf{9}$ were washed at least 10 times by centrifugation with ultrapure water to remove water soluble unreacted phosphotungstate species, then freeze-dried for 1 day. The final samples were kept at $20^{\circ} \mathrm{C}$ before any characterization and measurements.

2.3. Characterization of POM-based silica twisted nanoribbons (L)-8 and (D)-8 as well as helical nanoribbons (L)-9 and (D)-9.

\subsubsection{Solid ${ }^{31} P$ NMR measurements.}

${ }^{1} \mathrm{H}$ NMR spectra were recorded at $25^{\circ} \mathrm{C}$ with a Bruker Avance $300 \mathrm{FT}$ spectrometer $\left({ }^{1} \mathrm{H}\right.$ : $300 \mathrm{MHz}$ ) at IECB (Bordeaux, France). All proton chemical shifts are referenced to $\mathrm{Me}_{4} \mathrm{Si}$ 
(TMS). Solid ${ }^{31} \mathrm{P}$ NMR spectra were recorded on an Avance III NMR spectrometer (Bruker Biospin) with a vertical $11,74 \mathrm{~T}$ wide bore magnet operating at $202.47 \mathrm{MHz}$ for ${ }^{31} \mathrm{P}$ observation by means of a CP-MAS $4-\mathrm{mm} \mathrm{H} / \mathrm{X}$ probe. ${ }^{31} \mathrm{P}$ chemical shifts are reported relative to the external reference of ammonium dihydrogen phosphate (ADP) whose phosphorus group was set at $0 \mathrm{ppm}$. Samples were packed in $4 \mathrm{~mm} \mathrm{ZrO}_{2}$ CP-MAS rotors. Data processing was performed with Bruker TOPSPIN 3.5 software. All NMR experiments were recorded at $298 \mathrm{~K}$ at the spinning speed of $11 \mathrm{kHz}$, by means of a single pulse sequence with the following parameters : $15^{\circ}$ flip angle, sweep width of $59.5 \mathrm{kHz}$, a recycling delay of $2 \mathrm{~s}$, number of scans were adapted depending on the signal to noise ratio (between 642 and 88800 scans).

\subsubsection{UV-Vis Measurements}

The UV-Vis spectra were recorded with a Jasco J-815 CD spectrometer between 180 and $400 \mathrm{~nm}$, with $0.5 \mathrm{~nm}$ data interval and $200 \mathrm{~nm} / \mathrm{min}$ scan rate for the Venturello POM-Arquad and $20 \mathrm{~nm} / \mathrm{min}$ for the silica NANOPOM twisted ribbons $(\boldsymbol{L})-\mathbf{8}$ and $(\boldsymbol{D})-\mathbf{8}$ as well as helical nanoribbons $(\boldsymbol{L})-9$ and $(\boldsymbol{D})-9$ with a concentration of $0.2 \mathrm{mg} / \mathrm{ml}$. Quartz cuvette with optical path length $1 \mathrm{~cm}$ was used for the measurements.

\subsubsection{Raman measurements}

Raman spectra were recorded with a LabRam HR800 confocal spectrometer (HORIBA), using for excitation $514.5 \mathrm{~nm}$ radiation from an argon ion laser and equipped with an aircooled CCD detector (ANDOR) cooled to $-85^{\circ} \mathrm{C}$. The laser power at the samples was $10 \mathrm{~mW}$ and the spectra were accumulated 4 times during $900 \mathrm{~s}$. The spectral resolution given by the entrance slit of $100 \mu \mathrm{m}$ and the 600 lines $/ \mathrm{mm}$ grating was $6.8 \mathrm{~cm}^{-1}$. A DuoScan device composed of two mirrors was activated in order to prevent any sample degradation. Using this device, the excitation beam scan a $10 \times 10 \mu \mathrm{m}$ sized area with a 50x objective (NA 0.75) in the confocal regime. The Raman spectra were recorded between 200 and $1600 \mathrm{~cm}^{-1}$.

\subsubsection{Transmission electron microscopy (TEM) measurements}

TEM measurements were performed at room temperature on a Philips EM 120 electron microscope operating at $120 \mathrm{kV}$, and the images were collected by $2 \mathrm{k} \times 2 \mathrm{k}$ Gatan ssCCD camera. Drops of diluted dispersion of the hybrids were deposited on a carbon-coated 200/400-mesh copper grids. Hydrophilic grids were prepared for the observation of samples dispersed in water by treating the carbon-coated copper grids in BioForce Nanosciences 
UV/Ozone ProCleaner 220 for $20 \mathrm{~min}$. For the sample preparation, $5 \mu \mathrm{L}$ of silica suspension were drop-casted onto the grid and blotted after $1 \mathrm{~min}$. Samples were dried in air.

\subsubsection{HR-TEM measurements}

POM clusters grafted on the silica nanohelices were observed by a JEOL 2200FS high resolution transmission electron microscope operating at $200 \mathrm{kV}$ and several images was taken using the STEM mode with probe size: spot $1.5 \mathrm{~nm}$. EDX (Energy Dispersive X-ray Spectrometry) and element mapping were performed on the JEOL 2200FS using STEM mode.

\subsubsection{Elemental energy dispersive X-ray analysis (EDX)}

The EDX was used to estimate the quantity of POM grafted on the silica nanohelices. The atomic percentage of the tungsten $\mathrm{W}(\mathrm{L})$ and silicon $\mathrm{Si}(\mathrm{K})$, in the different samples was estimated by EDX. For each sample several areas of the sample were studied and the average of percentage of tungsten and silicon was calculated. $\%\left[\mathrm{PW}_{4} \mathrm{O}_{24}\right]^{3-}=\% \mathrm{~W} / 4 ; \%$ Silica $=\% \mathrm{Si}$. To obtain the $\%$ of POM in weight, the molar percentage of POM cluster was multiplied by its molar mass.

\subsubsection{Circular Dichroism measurements}

Electronic circular dichroism (ECD) measurements were performed on a Jasco J-815 CD spectrometer. The scan rate was $20 \mathrm{~nm} / \mathrm{min}$ and 50 scans were applied to each sample. The concentration of hybrids applied for these experiments was $0.2 \mathrm{mg} / \mathrm{ml}$. All CD experiments were carried out in acetonitrile with a quartz cuvette $\left(1 \mathrm{~cm}\right.$ path length) at $25^{\circ} \mathrm{C}$ and with stirring. The baseline corrections of the ECD spectra were performed by subtracting the two opposite-enantiomers ECD spectra and divided by two in case of APTES functionalized silica twisted nanoribbons (L)-6 and $(D)-6$ as well as helical nanoribbons $(L)-7$ and $(D)-7$ respectively. This baseline procedure removes the experimental artefacts coming from the ECD spectrometer. For the NANOPOM ribbons $(L)-\mathbf{8}$ and $(D)-\mathbf{8}$ as well as $(L)-\mathbf{9}$ and $(D)-\mathbf{9}$, the ECD spectra were subtracted from the ECD spectra of the APTES functionalized silica nanoribbons.

2.4. General procedure for catalytic oxidation reactions with POM-based silica twisted nanoribbons (L)-8 and (D)-8 and helical nanoribbons (L)-9 and (D)-9. 
Silica NANOPOM catalyst was dispersed in $1 \mathrm{~mL}$ of solvent and the sulfide ( 250 equiv) was added to the mixture. An aqueous solution of $\mathrm{H}_{2} \mathrm{O}_{2}$ (35\% in water, 375 equiv) was added to the reaction mixture at the appropriate temperature. The latter was stirred and monitored by ${ }^{1} \mathrm{H}$ NMR spectroscopy. Upon completion of the reaction, the catalyst was separated from the medium by centrifugation. The enantiomeric excess were evaluated by chiral HPLC using Chiracel ASH column and UV detector (254 nm), eluting with hexane/2-propanol (1:1) at a flow of $0.5 \mathrm{~mL} \cdot \mathrm{min}^{-1}$. Retention time: $(R)-\mathbf{1 1}$ : $22.5 \mathrm{~min}$; $(S)$-11: $30.9 \mathrm{~min}$.

\section{Results and discussion}

\subsection{Synthesis of peroxophosphotungstate-based silica twisted and helical nanoribbons.}

As summarized in Scheme 1, silica peroxophosphotungstate-based twisted $(L)-\mathbf{8}$ and $(D)-8$, and helical (L)-9 and (D)-9 nanoribbons were obtained by the treatment of amine-modified silica twisted (L)-6 and (D)-6), and helical nanoribbons $(L)-7$ and $(D)-7$ with the Keggin POM anion $\left[\mathrm{PW}_{12} \mathrm{O}_{40}\right]^{3-}$ and excess $\mathrm{H}_{2} \mathrm{O}_{2}[15]$.

\section{Scheme 1. (HERE)}

Schematic representation of the synthesis of right-handed POM-based silica twisted $(L)-\mathbf{8}$ and helical $(L)-9$ nanoribbons.

The amine functionalized silica twisted and helical nanoribbons used as chiral source for achiral POM clusters were prepared by the self-assembly of cationic bis-quaternary ammonium gemini surfactants $\quad\left[\left(N, N^{\prime}\right.\right.$-dihexadecane- $N, N, N^{\prime}, N^{\prime}$-tetramethyl $)$ ethylene diammonium] $(L)-\mathbf{1}$ and $(D)-\mathbf{1}$, noted here after as 16-2-16, according to the procedures described in references [16-17]. Depending on gel aging time, twisted ribbons $(L)-\mathbf{2}$ and $(D)-2$ with periodicity around $100 \mathrm{~nm}$ were obtained after $2 \mathrm{~h}$, whereas helical nanoribbons $(L)-3$ and $(D)-3$ with periodicity around $63 \mathrm{~nm}$ were obtained after 2 days. Right-handed helices are formed with $(L)$-tartrate whereas left-handed helices are obtained with $(D)$-tartrate counter anion . Organic twisted nanoribbons $(L)-\mathbf{2}$ and $(D)-\mathbf{2}$ as well as helical nanoribbons $(L)-\mathbf{3}$ and (D)-3 were used as template for the sol-gel condensation of tetraethyl orthosilicate (TEOS) to obtain the corresponding inorganic silica counterparts $(L)-\mathbf{4}$ and $(D)-\mathbf{4}$, as well as $(L)-\mathbf{5}$ and $(D)-5$, respectively. The latter react with APTES to give twisted nanoribbons $(L)-\mathbf{6}$ and $(D)-\mathbf{6}$, as well as helical nanoribbons $(L)-7$ and $(D)-7$, respectively. To build the peroxophosphotungstate salts of silica nanoribbons 6 and 7, we have used the procedure involving peroxide-mediated decomposition of the heteropolyacid $\mathrm{H}_{3} \mathrm{PW}_{12} \mathrm{O}_{40}$ [18]. According to this procedure, $\mathrm{H}_{3} \mathrm{PW}_{12} \mathrm{O}_{40}$ was decomposed in the presence of excess $\mathrm{H}_{2} \mathrm{O}_{2}$ to 
form the trianionic peroxophosphotungstate $\left[\mathrm{PO}_{4}\left(\mathrm{WO}\left(\mathrm{O}_{2}\right)_{2}\right)_{4}\right]^{3-}$ and the dinuclear peroxotungstate $\left[\left\{\mathrm{WO}\left(\mathrm{O}_{2}\right)_{2}\left(\mathrm{H}_{2} \mathrm{O}\right)\right\}_{2} \mathrm{O}\right]^{2-}$. It is well known that in a biphasic medium of water and organic solvant, the trianion $\left[\mathrm{PO}_{4}\left(\mathrm{WO}\left(\mathrm{O}_{2}\right)_{2}\right)_{4}\right]^{3-}$ reacts selectively with the ammonium moieties and transfered to the organic phase (phase-transfer agents), whereas $\left[\left\{\mathrm{WO}\left(\mathrm{O}_{2}\right)_{2}\left(\mathrm{H}_{2} \mathrm{O}\right)\right\}_{2} \mathrm{O}\right]^{2-}$ remains in the aqueous phase. In the work described herein, the trianionic peroxophosphotungstate $\left[\mathrm{PO}_{4}\left(\mathrm{WO}\left(\mathrm{O}_{2}\right)_{2}\right)_{4}\right]^{3-}$ is expected to react with ammonium groups of silica nanoribbons $\mathbf{6}$ and $\mathbf{7}$ to give silica twisted and helical nanoribbons $\mathbf{8}$ and $\mathbf{9}$, which contain the peroxophosphotungstate within their structures. The dinuclear $\left[\left\{\mathrm{WO}\left(\mathrm{O}_{2}\right)_{2}\left(\mathrm{H}_{2} \mathrm{O}\right)\right\}_{2} \mathrm{O}\right]^{2-}$ and others non identified POM species were removed from water supernatant by several washing and centrifugation steps.

\subsection{Characterization of silica NANOPOM hybrids.}

The morphology and the structure of the resulting NANOPOM twisted ribbons $(L)-\mathbf{8}$ and $(D)$ $\mathbf{8}$ as well as helical ribbons $(L)-\mathbf{9}$ and $(D)-\mathbf{9}$ were characterized by TEM, HR-TEM, STEM, EDX, UV-Vis and Raman. Particular focus was devoted to solid state ${ }^{31} \mathrm{P}$ NMR and circular dichroism (CD) spectroscopy, as the ${ }^{31} \mathrm{P}$ NMR offers evidence for the presence of the peroxophosphotungstate species within NANOPOM hybrids, whereas the CD highlights the chirality induction to POM species.

\subsubsection{Solid ${ }^{31} P$ NMR measurements}

As shown in Figure SI1a in the supporting information, the ${ }^{31} \mathrm{P}$ NMR spectrum of NANOPOM twisted ribbons $(L)-8$ showed three broad peaks at 3.5, -6.1 and -14.7 ppm indicating a mixture of phosphorus-containing POM species. We were unable to obtain a better ${ }^{31} \mathrm{P}$ NMR spectrum with a sufficiently higher signal-to-noise ratio, probably because of the low amount of phosphorus-containing POM species within NANOPOM hybrids. However, according to the ${ }^{31} \mathrm{P}$ NMR spectrum, the presence of the peroxophosphotungstate $\left[\mathrm{PO}_{4}\left(\mathrm{WO}\left(\mathrm{O}_{2}\right)_{2}\right)_{4}\right]^{3-}$ is confirmed by the signal at $\delta=3.5 \mathrm{ppm}$. Interestingly, this value is comparable to that obtained at $\delta=2.2 \mathrm{ppm}$ for the solid ${ }^{31} \mathrm{P}$ NMR measurement of

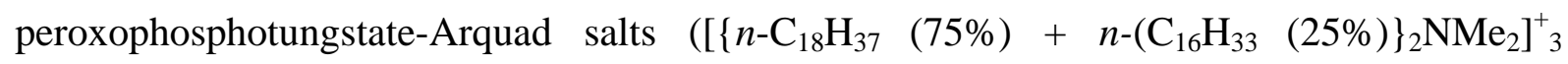
$\left.\left[\mathrm{PO}_{4}\left\{\mathrm{WO}\left(\mathrm{O}_{2}\right)_{2}\right\}_{4}\right]^{3-}\right)$, in which Arquad $=\left(\left[\left\{n-\mathrm{C}_{18} \mathrm{H}_{37}(75 \%)+n-\left(\mathrm{C}_{16} \mathrm{H}_{33}(25 \%)\right\}_{2} \mathrm{NMe}_{2}\right]\right.\right.$ (Figure SI1b in Supporting Information).In addition, Hill [18c] and other groups [19] have shown that $\left[\mathrm{PO}_{4}\left(\mathrm{WO}\left(\mathrm{O}_{2}\right)_{2}\right)_{4}\right]^{3-}$ can evolve to a mixture of phosphorus-contaning species in acidic mediums, depending on the system and the experimental conditions. For example in a 
biphasic medium of water and dichlorometane, only one signal was obtained in the ${ }^{31} \mathrm{P}$ NMR of $\left[\mathrm{PO}_{4}\left(\mathrm{WO}\left(\mathrm{O}_{2}\right)_{2}\right)_{4}\right]^{3-}$ containing POM-cored dendrimers $[4 \mathrm{c}-\mathrm{h}]$. In this case, the peroxophosphotungstate $\left[\mathrm{PO}_{4}\left(\mathrm{WO}\left(\mathrm{O}_{2}\right)_{2}\right)_{4}\right]^{3-}$ is protected against the acidic attacks by the dendritic structure around the POM species, as well as its transfer to the organic phase which prevents its transformation to new phosphotungstate species. In the system described herein, the presence of a mixture of phosphorus-containing species within the structure of NANOPOM hybrids can be explained by the slow transformation of the peroxophosphotungstate trianion to new phosphorus-containing species in acidic reaction medium, due to acidic attacks. Thus, according to the litterature, the broad peaks obtained in the ${ }^{31} \mathrm{P}$ NMR spectrum, at 3.5, -6.1 and $-14.7 \mathrm{ppm}$ should be assigned to $\left[\mathrm{PO}_{4}\left(\mathrm{WO}\left(\mathrm{O}_{2}\right)_{2}\right)_{4}\right]^{3-}$ Venturello anion, an undefined lacunary Keggin-type structure and the $\left[\mathrm{PW}_{12} \mathrm{O}_{40}\right]^{3-}$ Keggin species, respectively.

\subsubsection{TEM, HR-TEM, STEM and EDX analyses measurements}

Transmission Electron Microscopy (TEM) measurements were performed for all the silica nanoribbons summarized in scheme 1 . The twisted and helical morphologies are evidenced for POM-based nanoribbons $(L)-\mathbf{8}$ and $(L)-\mathbf{9}$ as shown in Fig. 1a and Fig. 1b, respectively.

Fig.1. (HERE)

TEM images of silica NANOPOM: a) POM with silica twisted ribbons $(L)-\mathbf{8}$ and b) POM with silica helical ribbons $(L)-9$; HR-TEM images of silica NANOPOM: c) Silica twisted ribbons ( $L$ )-8 and d) Silica helical ribbons (L)-9; STEM images of silica NANOPOM: e) Silica twisted ribbons $(L)-8$ and f) Silica helical ribbons $(L)-9$.

No discernible change was observed by the TEM images in the morphology, size and periodicity of POM-based nanoribbons $(L)-\mathbf{8}$ and $(L)-\mathbf{9}$, with respect to those obtained for APTES functionalized silica twisted $(L)-6$ and helical $(L)-7$ nanoribbons, as stated in Supporting Information (Fig. SI2a and SI2b). Indeed, well defined NANOPOM hybrids with the diameter of $18 \pm 2 \mathrm{~nm}$ for twisted ribbons and the diameter of $29 \pm 4 \mathrm{~nm}$ for helical ribbons and pitches of $95 \pm 3 \mathrm{~nm}$ for twisted ribbons and $64 \pm 5 \mathrm{~nm}$ for helical ribbons were obtained. All these are in good agreement with the silica twisted or helical ribbons before the POM grafting, indicating a high stability of these silica nanoribbons during the POM grafting.

Interestingly, the high-resolution HR-TEM images of silica POM-based twisted ribbons (L)-8 (Figure 1c) and POM-based helical ribbons (L)-9 (Figure 1d) as well as their scanning transmission electron microscopy (STEM) shown in Fig. 1e and 1f, confirm the uniformity of 
these NANOPOM hybrids. Black dots attributed to phosphotungstate anions are observed within the nanoribbons. The diameter sizes of POM clusters in the range of 0.7 to $1.3 \mathrm{~nm}$ are consistent with that of the peroxophosphotungstate trianion alone or as small aggregates. HRTEM and STEM images of NANOPOM ribbons revealed that for both nanoribbons, POMs are organized on the silica surface, without strong aggregation, as already observed in our previous work, for electrostatic grafting of the Keggin anion [10]. Interestingly, compared to helical ribbons $(L)-\mathbf{9}$, the surface of silica twisted ribbons $(L)-\mathbf{8}$ showed more black dots, indicating higher concentration of POM clusters.

In order to confirm the presence of POM clusters within silica nanoribbons, NANOPOM hybrids were characterized by elemental energy dispersive X-ray analysis (EDX). The EDX analysis at several locations on the surface of nanoribbons showed comparable results. The spectra of silica twisted nanoribbons (L)-8 (Fig. 2a) and helical nanoribbons (L)-9 (Fig. 2b) reveal the presence of tungsten element, indicating a successful incorporation of phosphotungstate anions within the structure of silica twisted and helical nanoribbons, respectively.

Fig.2. (HERE)

EDX spectra of silica NANOPOM: a) twisted ribbons $(L)-\mathbf{8}$ and b) helical ribbons $(L)-\mathbf{9}$.

Even if silicon was the dominant element, tungsten was also detected as shown in Figure 3. Based on these results, it is demonstrated that phosphotungstate clusters were successfully immobilized onto the surface of silica nanoribbons, without altering their morphologies. The average of atomic percentage of tungsten was found as $4.75 \% \%$ and $3.24 \%$ for twisted ribbons $(L)-\mathbf{8}$ and helical nanoribbons $(L)-\mathbf{9}$, respectively. These results are in agreement with the HR-TEM images (Figure 1), that showed a higher concentration of POM clusters on twisted ribbon hybrids.

\section{Fig.3. (HERE)}

a) STEM images of silica helical nanoribbons $(L)-9$; b) Elemental mappings of tungsten (W) and silicon ( $\mathrm{Si})$; c) Elemental mapping of $\mathrm{Si}$; d) Elemental mapping of $\mathrm{W}$.

As shown in Fig.3a to 3d, the elemental mappings patterns of silica helical nanoribbons $(L)$ 9 confirm that the black dots correspond to phosphotungstate anions. We have estimated the 
amount of POM within silica nanoribbons and found average values of $20 \mathrm{w} \%$ and $14 \mathrm{w} \%$ for silica twisted ribbons 8 and helical ribbons $\mathbf{9}$, respectively.

\subsubsection{Raman spectroscopy of NANOPOM hybrids.}

The grafting of POM clusters onto silica nanoribbons was also investigated using Raman spectroscopy. The Raman spectrum of NANOPOM twisted ribbons 8 (Fig. 4) exhibits the two most intense bands characteristic of the peroxophosphotungstate $\left[\mathrm{PO}_{4}\left(\mathrm{WO}\left(\mathrm{O}_{2}\right)_{2}\right)_{4}\right]^{3-}$ trianion at $958 \mathrm{~cm}^{-1}$ and $220 \mathrm{~cm}^{-1}$, attributed to the stretching $\mathrm{W}=\mathrm{O}$ and bending WOW vibrations, respectively [11d,20]. The band observed at $490 \mathrm{~cm}^{-1}$ can be assigned to bending vibration of Si-O-Si groups [21], whereas the small bands in the $1460-1410 \mathrm{~cm}^{-1}$ spectral range are related to APTES. The Raman spectrum of NANOPOM helical ribbons 9 reveals comparable bands to those of twisted ribbons $\mathbf{8}$. Nevertheless, the $\mathrm{W}=\mathrm{O}$ band is observed at higher wavenumbers (ca $970 \mathrm{~cm}^{-1}$ ) whereas the shoulder at $1040 \mathrm{~cm}^{-1}$ is slightly more intense than for twisted ribbons. The positions of the $\mathrm{W}=\mathrm{O}$ band for $(L)-8$ and $(L)-\mathbf{9}$ are different to the one observed for the Keggin heteropolyacid $\mathrm{H}_{3} \mathrm{PW}_{12} \mathrm{O}_{40}$ grafted onto the silica nanohelices (ca $980 \mathrm{~cm}^{-1}$ ) [10]. This slight shift observed in the case of NANOPOMs 8 and 9 as well as the presence of a shoulder at $1040 \mathrm{~cm}^{-1}$ may resulted on the presence of a phosphotungstate mixture, instead of the Keggin or the peroxophosphotungstate species alone. Finally, the ratio of the intensity of the $v \mathrm{~W}=\mathrm{O}$ and $v \mathrm{Si}-\mathrm{O}-\mathrm{Si}$ bands is clearly higher for $(L)-\mathbf{8}$ than for $(L)-\mathbf{9}$, confirming the higher concentration of POM clusters on twisted ribbon hybrids.

\section{Fig.4. (HERE)}

Raman spectra of silica twisted ribbons $(L)$-8 (black spectrum), silica helical ribbons $(L)-\mathbf{9}$ (red spectrum), and Keggin heteropolyacid immobilized onto $(L)-7$ (blue spectrum).

3.2.4. UV-Vis measurements and chiroptical properties of NANOPOM twisted ribbons $(L)$ 8, (D)-8 and helical ribbons (L)-9, (D)-9..

The UV-Vis measurement of twisted ribbons $(L)-8,(D)-8$ and helical ribbons $(L)-\mathbf{9},(D)-\mathbf{9}$ have been performed in acetonitrile. The spectra of twisted nanoribbons $(L)-\mathbf{8}$ (or $(D)-\mathbf{8})$ (Fig. 5a) shows an intense absorption band around $200 \mathrm{~nm}$ and a weak band around $260 \mathrm{~nm}$, which correspond to the $\mathrm{O} \rightarrow \mathrm{W}$ charge-transfer band of phosphotungstate [4f, 19b, 22]. As expected, a UV signal characteristic to phosphotungstate absorption was observed, indicating the presence of phosphotungstate species within silica nanoribbons. Interestingly, these signals are comparable to those obtained for the UV-Vis measurement of 


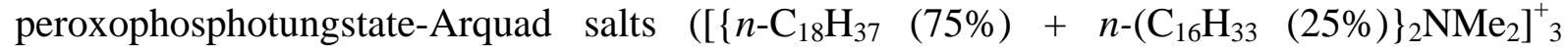
$\left.\left[\mathrm{PO}_{4}\left\{\mathrm{WO}\left(\mathrm{O}_{2}\right)_{2}\right\}_{4}\right]^{3-}\right)$, in which Arquad $=\left(\left[\left\{n-\mathrm{C}_{18} \mathrm{H}_{37}(75 \%)+n-\left(\mathrm{C}_{16} \mathrm{H}_{33}(25 \%)\right\}_{2} \mathrm{NMe}_{2}\right]\right.\right.$ (Figure SI3 in Supporting Information) [18c].

\section{Fig.5. (HERE)}

(a) UV-Vis spectra of silica NANOPOM twisted ribbons $(L)-8$ or $((D)-8)$ in acetonitrile; (b) CD spectra of APTES functionalized silica twisted ribbons before $(L)-6,(D)-\mathbf{6}$, and after mixing with POM $(L)-8,(D)-8$ in acetonitrile.

The chiroptical activity of NANOPOM twisted ribbons $(L)-\mathbf{8},(D)-\mathbf{8}$ and the corresponding helical ribbons $(L)-\mathbf{9},(D)-\mathbf{9}$, has been investigated by recording their circular dichroism (CD) spectra in aqueous suspensions. As shown in fig. 5b, the CD spectra of silica twisted ribbons $(L)-8$ and $(D)-8$ presents opposite cotton effects, confirming the reverse chirality between the left- and right-handed ribbons. These CD signals are comparable to those recently obtained for silica NANOPOM built from electrostatic grafting of Keggin trianion onto silica nanohelices [10]. As expected, no CD signal was obtained for APTES-functionalized silica twisted ribbons $(L)-6$ and $(D)-6$ before the grafting of POM clusters. Thus, the CD signal obtained for NANOPOMs $(L)-\mathbf{8}$ and $(D)-\mathbf{8}$ are attributed to the induced chirality to POM clusters, highlighting a chiral induction ability of twisted ribbons within NANOPOM hybrids.

In order to evaluate the effects of the structure of nanoribbons (twisted versus helical structures) on the induced chirality to POM, we have compared the CD signals of helical NANOPOM hybrids $(L)-\mathbf{9}$ and $(D)-\mathbf{9}$ with those of twisted counterparts $(L)-\mathbf{8}$ and $(D)-\mathbf{8}$, in acetonitrile. Helical NANOPOM hybrids $(L)-9$ and $(D)-9$ showed less significant induced circular dichroism (ICD) than twisted ribbons $(L)-\mathbf{8}$ and $(D)-\mathbf{8}$, as shown in Supporting Information (Fig. SI4b). These results may be explained by the higher amount of POM units within twisted NANOPOMs, and their organization within these structures, as indicated by EDX analyses and TEM images. These images showed a well arrangement of POM units following the pitch of the silica twisted ribbons. On the other hand, the weaker CD obtained for NANOPOM hybrids 8 and $\mathbf{9}$ with respect to silica NANOPOM built from direct adsorption of Keggin anion may result to the presence of the amino propyl spacer between POM units and nanoribbons (chiral sources), that increases the distance between the two components. In addition, the small size of peroxophosphotungstate anions $c a 0.7 \mathrm{~nm}$ may also contribute to the decrease of the chirality induction. To support this point, it has been reported for helical gold nanoparticle superstructures that increasing the diameter of nanoparticles decrease the interparticle distance, resulting to the increase of the $\mathrm{CD}$ signals of the 
corresponding hybrid material [9a] Even if the induced chirality is not as intensive as for silica NANOPOM built from more stable Keggin anion, to the best of our knowledge, the systems described herein represent the first report in which achiral peroxophosphotungstate anions have been immobilized onto twisted and helical nanoribbons and display chiroptical effects, as a result of the chirality induction.

\subsection{Catalytic tests of phosphotungstate-based silica twisted ribbons $\mathbf{8}$ and helical ribbons $\mathbf{9}$} in the oxidation of methyl phenyl sulfide 10.

We tested the catalytic performance of NANOPOM twisted $(L)-8$ and helical ribbons $(L)-\mathbf{9}$ in the oxidation of methyl phenyl sulfide 10 in $\mathrm{CH}_{3} \mathrm{CN}$ and acetone, using $\mathrm{H}_{2} \mathrm{O}_{2}(35 \%)$ as primary oxidant. The reaction was monitored over time by plotting the ratio between the intensity of the disappearing ${ }^{1} \mathrm{H}$ signals of sulfide $\mathbf{1 0}$ and the new peaks of the product. The results summarized in Table 1 showed that the NANOPOM hybrids $(L)-\mathbf{8}$ and $(L)-\mathbf{9}$ catalyzed the oxidation of methyl phenyl sulfide to the corresponding methyl phenyl sulfoxide $\mathbf{1 1}$ in a racemic form and methyl phenyl sulfone $\mathbf{1 2}$.

\section{Table 1 (HERE)}

Oxidation of methyl phenyl sulfide $\mathbf{1 0}$ catalyzed by silica NANOPOM hybrids $\mathbf{8}$ and $\mathbf{9}$, using $\mathrm{H}_{2} \mathrm{O}_{2} \cdot{ }^{\mathrm{a}}$

The selectivity to sulfoxide increased with decreasing reaction temperature. As shown in Table 1, in the presence of NANOPOM twisted ribbon $(L)-\mathbf{8}$, methyl phenyl sulfone $\mathbf{1 0}$ is oxidized in $\mathrm{CH}_{3} \mathrm{CN}$ with $100 \%$ conversion after 30 min, giving $89 \%$ sulfoxide 11 and $11 \%$ of sulfone 12 , at $-30^{\circ} \mathrm{C}$ (Table 1, entry 1), whereas $68 \%$ of sulfoxide $\mathbf{1 1}$ and $32 \%$ of sulfone $\mathbf{1 2}$ were obtained after $15 \mathrm{~min}$ at $0^{\circ} \mathrm{C}$ (entry 3 ). In similar condition, NANOPOM helical ribbon (L)-9 oxidize methyl phenyl sulfide 10 with $100 \%$ conversion after $30 \mathrm{~min}$ at $0^{\circ} \mathrm{C}$ (entry 6), indicating that NANOPOM twisted ribbon $(L)-\mathbf{8}$ is the more efficient catalytic system in the conditions used. This result may be due to the higher concentration of phosphotungstate anions within twisted ribbons than their helical ribbons analogues.

Compare to silica nanohelices based on the Keggin trianion $\left[\mathrm{PW}_{12} \mathrm{O}_{40}\right]$ reported recently [10], the oxidation of methyl phenyl sulfide $\mathbf{1 0}$ with peroxophosphotungstate-based nanoribbons 8 and 9 indicate a significant increase in the reaction kinetic, whereas the selectivity to the sulfoxide product decreases. These results are in agreement with those reported for peroxophosphotungstate catalysts and highlight the transformation of the 
Keggin's POM [ $\left.\mathrm{PW}_{12} \mathrm{O}_{40}\right]$ into phosphotungstate species [4c-h, 18c]. It has been shown that under similar conditions, the peroxophosphotungstate anion is a more active oxidation catalyst than the Keggin species, but can be quickly disable depending on the counter ion and the microenvironment around. Thus, this catalytic system can be defined as a mixture of phosphotungstate anions which also contains the peroxophosphotungstate species. It is interesting to note that without POM, less than $4 \%$ conversion of sulfide to sulfoxide was generally obtained after $6 \mathrm{~h}$ under similar conditions. Reactions in acetone (entries 4-5) are less efficient that those in $\mathrm{CH}_{3} \mathrm{CN}$. The evaluation of the optical properties of twisted ribbons and helical ribbons in the oxidation of sulfide $\mathbf{1 0}$ indicates no significant enantioselectivity (ee) even at low temperature. Even if a CD signal which reflects a chirality induction on the POM is obtained for twisted nanoribbons $\mathbf{8}$, this property is not expressed at the level of the oxidation reaction of methyl phenyl sulfide 10. We believe that new approaches for the preparation of helical NANOPOM hybrids with greater potential for chiral induction, such as a covalent coupling of POM units with nanohelices, should be interesting in promoting the enantioselectivity. The development of catalytic systems for this purpose is in progress.

\section{Conclusions}

In the quest for new chiral POM-based nanomaterials, we have developed a simple way to prepare peroxophosphotungstate-based silica twisted and helical nanoribbons by coupling achiral peroxophosphotungstate anions with well defined amine-funtionalized twisted and helical nanoribbons in acidic medium. The structures of these silica NANOPOM hybrids were determined by ${ }^{31} \mathrm{P}$ NMR, TEM and HR-TEM as well as UV-Vis and Raman spectroscopy. The induced chirality of POM was demonstrated by electronic circular dichroism measurements which showed a higher CD signal for silica twisted ribbons with respect to silica helical ribbons, due to the higher amount of POM clusters and their arrangement onto the chiral source. Compared to silica NANOPOM helices based on the Keggin anion previously reported, the amount of phosphotungstate grafted onto the silica matrix is lower, probably due to the difference in stability between the two POM species, the Keggin anion being the most stable. However, as expected, under comparable conditions, silica NANOPOM hybrids based on the peroxophosphotungstate are more active catalysts in the oxidation of mehyl phenyl sulfide. We expect to improve the stability and the chiroptical properties of these new systems by isolating the peroxophosphotungstate anion before its grafting on the silica matrix and its direct adsorption on the chiral sources. We believe that an 
increase in the stability of the peroxophosphotungstate cluster added to its proximity to the chiral source should improve the chiroptical properties of resulting NANOPOMs. Further studies with this approach are in progress.

\section{Acknowledgements}

Financial support from the University of Bordeaux and the CNRS is gratefully knowledged. We thank IECB technology platforms and S. Tan, M. Decossas and E. Morvan, A. Grelard (CBMN) for TEM and ${ }^{31} \mathrm{P}$ NMR experiments, S. Buffière (ICMCB, CNRS) and Placamat platform for HR-TEM and EDX measurements.

\section{References}

[1] a) M. T. Pope, A. Müller, Angew. Chem.Int. Ed. Engl. 1991, 30, 34-48; b) J. T. Rhule, C. L. Hill, D. A. Rud, R. F. Schinazi, Chem. Rev. 1998, 98, 327-358; c) Dimitris E. Katsoulis, Chem. Rev. 1998, 98, 359-388; d) M. T. Pope, A. Müller, Polyoxometalate Chemistry: From Topology via Self-Assembly to Applications, Kluwer Academic, Dordrecht, 2001.

[2] a) Chem. Rev. Special Thematic Issues, Polyoxometalates. 1998, 98, 1-389; b) A. Proust, R. Thouvenot, P. Gouzerh, Chem. Commun. 2008, 16, 1837-1852; c) D. L. Long, E. Burkholder, L. Cronin, Chem. Soc. Rev. 2007, 36, 105-121; d) D. Long, R. Tsunashima, L. Cronin, Angew. Chem. Int. Ed. 2010, 49, 1736-1758; e) P. Mialane, A. Dolbecq, F. Sécheresse, Chem. Commun. 2006, 33, 3477-3485; f) A. Dolbecq, E. Dumas, C. R. Mayer, P. Mialane, Chem. Rev. 2010, 110, 6009-6048; g) S. Nlate, C. Jahier, Eur. J. Inorg. Chem. 2013, 1606-1619.

[3] a) B. Hasenknopf, K. Micoine, E. Lacôte, S. Thorimbert, M. Malacria, R. Thouvenot, Eur. J. Inorg. Chem. 2008, 5001-5013. (and reference therein); b) D. A. J. Judd, H. Nettles, N. Nevins, J. P. Snyder, D. C. Liotta, J. Tang, J. Ermolieff, R. F. Schinazi, C. L. Hill, J. Am. Chem. Soc. 2001, 123, 886-887.

[4] a) H. Zeng, G. R. Newkome, C. L. Hill, Angew. Chem. Int. Ed. 2000, 39, 1771-1774; b) D. Volkmer, B. Bredenkötter, J. Tellenbröker, P. Kögerler, D. G. Kurth, P. Lehmann, H. Schnablegger, D. Schwahn, M. Piepenbrink, B. Krebs, J. Am. Chem. Soc. 2002, 124, 10489-10496; c) L. Plault, A. Hauseler, S. Nlate, D. Astruc, J. Ruiz, S. Gatard, R. Neumann, Angew. Chem. Int. Ed. 2004, 43, 2924-2928; d) S. Nlate, D. Astruc, R. Neumann, Adv. Synth. Catal. 2004, 346, 1445-1448; e) S. Nlate, L. Plault, D. Astruc, 
Chem. Eur. J. 2006, 12, 903-914; f) C. Jahier, M. Cantuel, N. D. McClenaghan, T. Buffeteau, D. Cavagnat, F. Agbossou, M. Carraro, M. Bonchio, S. Nlate, Chem. Eur. J. 2009, 15, 8703-8708; g) C. Jahier, M.-F. Coustou, M. Cantuel, N. D. McClenaghan, T. Buffeteau, D. Cavagnat, M. Carraro, S. Nlate, Eur. J. Inorg. Chem. 2011, 727-738; h) C. Jahier, S. Nlate, Eur. J. Inorg. Chem. 2012, 833-840.

[5] a) J. Zhang, W.-L. Chen, E. B. Wang, Inorg. Chem. Commun., 2013, 38, 96-99; b) A. Dolbecq, P. Mialane, B. Keita, L. Nadjo, J. Mater., Chem. 2012, 22, 24509-24521; b) W. Qi, Y. Wang, W. Li, L. Wu, Chem. Eur. J. 2010, 16, 1068-1078; c) W. Qi, H. Li, L. Wu, J. Phys. Chem. B. 2008, 112, 8257-8263; d) F. Shahbazi, K. Amani, Catal. Commun. 2014, 55, 57-64.

[6] a) M. Lu, J. H. Kang, D. G. Wang, Z. H. Peng, Inorg. Chem., 2005, 44, 7711-7713; b) M. Carraro, G. Modugno, A. Sartorel, G. Scorrano, M. Bonchio, Eur. J. Inorg. Chem, 2009, 34, 5164-5174; c) L. Yue, H. Ai, Y. Yang, W. Lu, L. Wu, Chem. Commun., 2013, 49, 9770-9772; d) B. Zhang, L. Yue, Y. Wang, Y. Yang, L. Wu, Chem. Commun., 2014, 50, 10823-10826; e) B. Zhang, W. Guan, F. Yin, J. Wang, B. Li, L. Wu, Dalton Trans., 2018, 1388-1392.

[7] a) Q. Tang, S. Liu, Y. Liu, S. Li, F. Ma, J. Li, S. Wang, C. Liu, Dalton Trans. 2013, 42, $8512-8518$; b) V. Kulikov, N. A. B. Johnson, A. J. Surman, M. Hutin, S. M. Kelly, M. Hezwani, D-L. Long, G. Meyer, L. Cronin, Angew. Chem. Int. Ed. 2017, 56, 1141-1145; c) J. Zhang, W. Li, C. Wu, B. Li, J. Zhang, L. Wu, Chem. Eur. J. 2013, 19, 8129-8135.

[8] A. Ben-Moshe, B. M. Maoz, A. O. Govorov, G. Markovich, Chem. Soc. Rev., 2013, 42, 7028-7041.

[9] a) J. Cheng, G. Le Saux, J. Gao, T. Buffeteau, Y. Battie, P. Barois, V. Ponsinet, M.-H. Delville, O. Ersen, E. Pouget, R. Oda, ACS Nano, 2017, 11, 3806-3818; b) R. Tamoto, S. Lecomte, S. Si, S. Moldovan, O. Ersen, M.-H. L. N. Delville, R. Oda., J. Phys. Chem. C 2012, 116, 23143-23152; c) S. H. Jung, J. Jeon, H. Kim, J. Jaworski, J. H. Jung. J. Am. Chem. Soc. 2014, 136, 6446-6452.

[10] M. Attoui, E. Pouget, R. Oda, D. Talaga, G. Le Bourdon, T. Buffetaeu, S. Nlate, , Chem. Eur. J. 2018, 24, 11344-11353.

[11] a) C. Venturello, R. D’Aloisio, J. C. J. Bart, M. Ricci, J. Mol. Catal. 1985, 32, 107-110; b) C. Venturello, R. D'aloisio J. Org. Chem. 1988, 53, 1553-1557; c) Y. Ishii, K. Yamawaki, T. Ura, H. Yamada, T. Yoshida, M. Ogawa, J. Org. Chem.1988, 53, 35873593; d) C. Aubry, G. Chottard, N. Platzer, J. M. Bregeault, R. Thouvenot, F. Chauveau, C. Huet, H. Ledon, Inorganic Chemistry 1991, 30, 4409-4415; e) Y. Ishii, M. Ogawa, 
Reviews on Heteroatom Chemistry, Vol. 3 (Eds: A. Ohno, N. Furukawa), MYU, Tokyo, 1990, p.121; f) R. Neumann, A. M. Khenkin, J. Org. Chem. 1994, 59, 7577-7579.

[12] S. Guha, M. G. Drew, A. Banerjee, small, 2008, 4, 1993-2005.

[13] J. Liu, L. Yang, Z. Huang, small, 2016, 12, 5902-5909.

[14] A. Brizard, D. Berthier, C. Aimé, T. Buffeteau, D. Cavagnat, L. Ducasse, I. Huc, R. Oda, Chirality 2009, 21, E153-E162.

[15] B. F. Sels, A. L. Villa, D. Hoegaerts, D. E. De Vos, P. A. Jacobs, Topic in catalysis, 2000, 13, 223-229.

[16] a) S. Manet, Y. Karpichev, D. Bassani, R. Kiagus-Ahmad, R. Oda, Langmuir 2010, 26, 10645-10656.

[17] T. Delclos, C. Aimé, E. Pouget, A. Brizard, I. Huc, M.-H. Delville, R. Oda, Nano. Lett. 2008, 8, 1929-1935.

[18] a) L. Salles, C. Aubry, R. Thouvenot, F. Robert, C. Doremieux-Morin, G. Chottard, H. Ledon, Y. Jeannin, J. M. Bregeault, Inorganic Chemistry 1994, 33, 871-878; b) A. J. Bailey, W. P. Griffith, B. C. Parkin, J. Chem. Soc. Dalton Trans. 1995, 1833-1837; c) D. C. Duncan, R. C. Chambers, E. Hecht, C. L. Hill, J. Am. Chem. Soc. 1995, 117, 681-691.

[19] a) L. Carlos de la Garza, K. De Oliveira Vigier, G. Chatel, A. Moores, Green Chem 2017, 2855-2862; b) W. Zhao, Y. Ding, Reac. Kinet. Mech. Cat., 2013, 109, 509-524.

[20] D. Hoegaerts, B. F. Sels, D. E. De Vos, F. Verpoort, P. A. Jacobs, Catalysis Today 2000, 60, 209-218.

[21] Y. Okazaki, T. Buffeteau, E. Siurdyban, D. Talaga, N. Ryu, R. Yagi, E. Pouget, M. Takafuji, H. Ihara, R. Oda, Nano Lett. 2016, 16, 6411-6415.

[22] a) H. Y. An, E. B. Wang, D. R. Xiao, Y. G. Li, Z. M. Su, L. Xu, Angew. Chem.Int. Ed. 2006, 45, 904-908; b) A Tézé, J. Canny, L. Gurban, R. Thouvenot, G. Hervé, Inorg. Chem. 1996, 35, 1001-1005. 


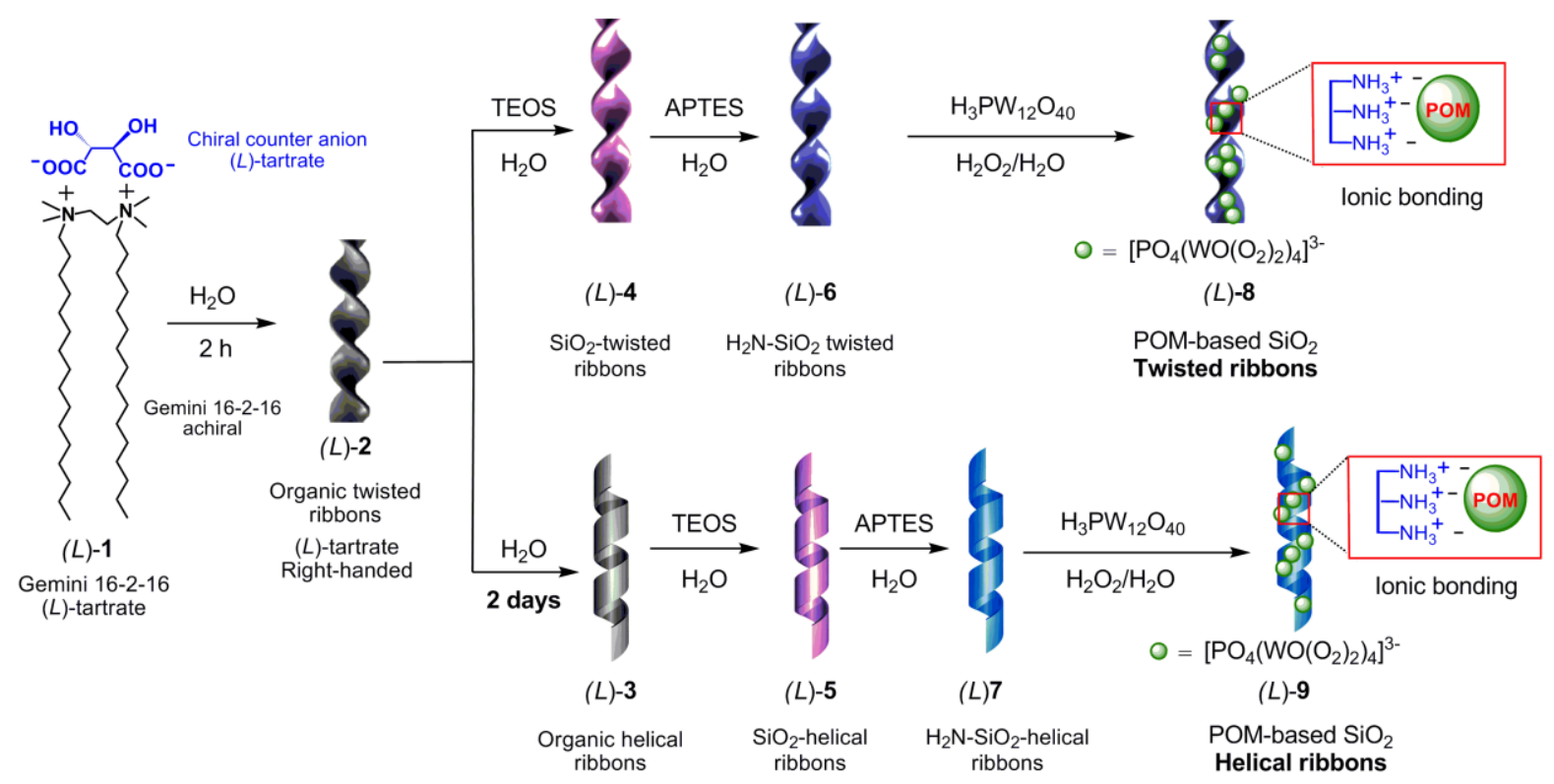

Silica POM-based helical and twiwted ribbons $(D)-8$ and $(D)-9$ were also prepared from Gemini 16-2-16 $(D)$-tartrate, $(D)$-1, using the same procedure

\section{Scheme 1.}

Schematic representation of the synthesis of right-handed POM-based silica twisted $(L)-8$ and helical $(L)-9$ nanoribbons. 
a)

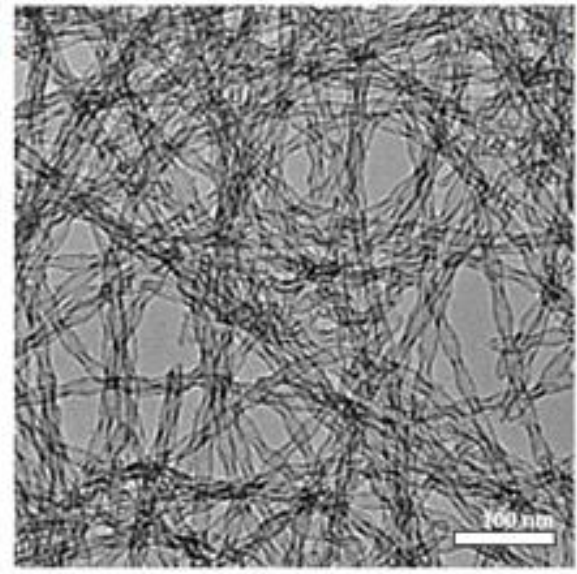

c)

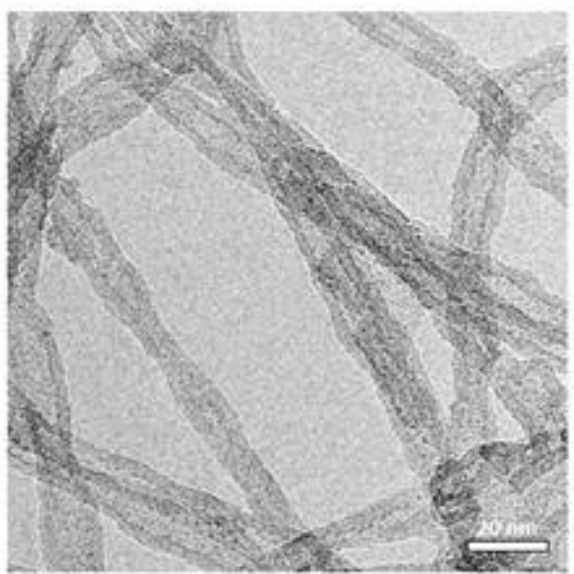

e)

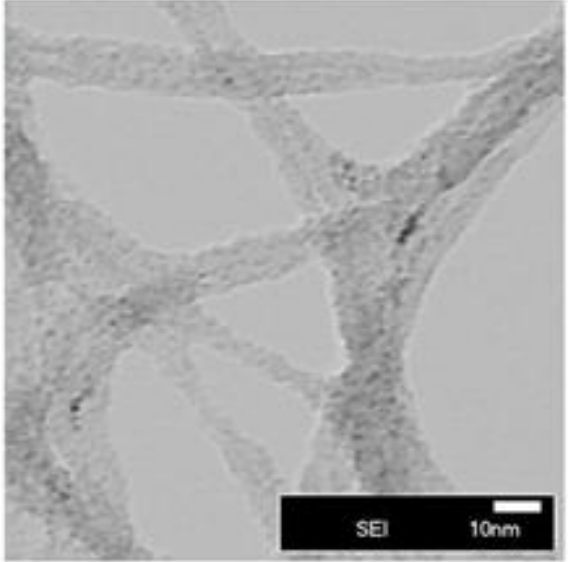

b)

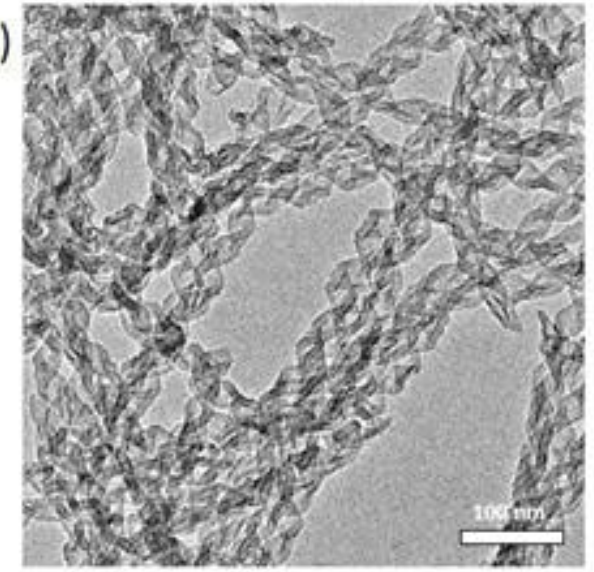

d)

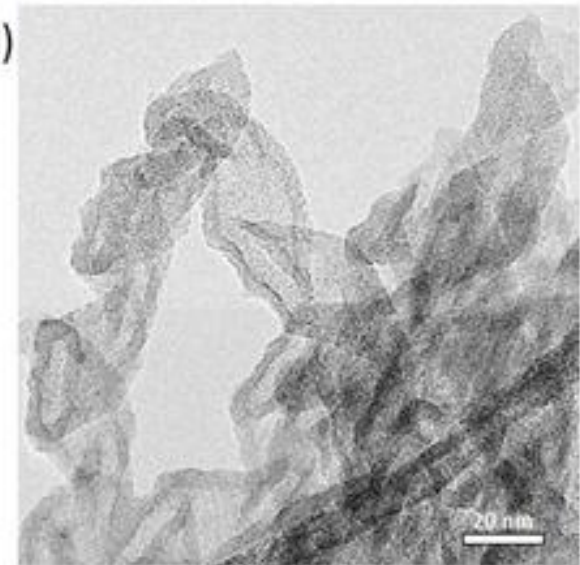

f)

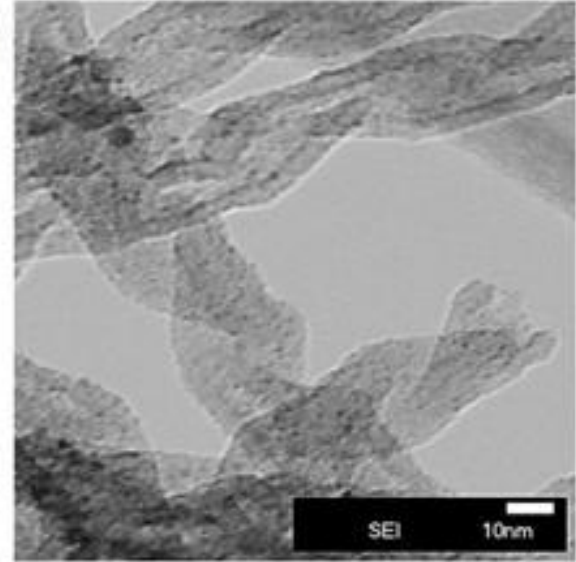

Fig.1.

TEM images of silica NANOPOM: a) POM with silica twisted ribbons $(L)-\mathbf{8}$ and b) POM with silica helical ribbons $(L)$-9; HR-TEM images of silica NANOPOM: c) Silica twisted ribbons $(L)-8$ and d) Silica helical ribbons ( $L)-9$; STEM images of silica NANOPOM: e) Silica twisted ribbons $(L)-8$ and f) Silica helical ribbons $(L)-\mathbf{9}$. 


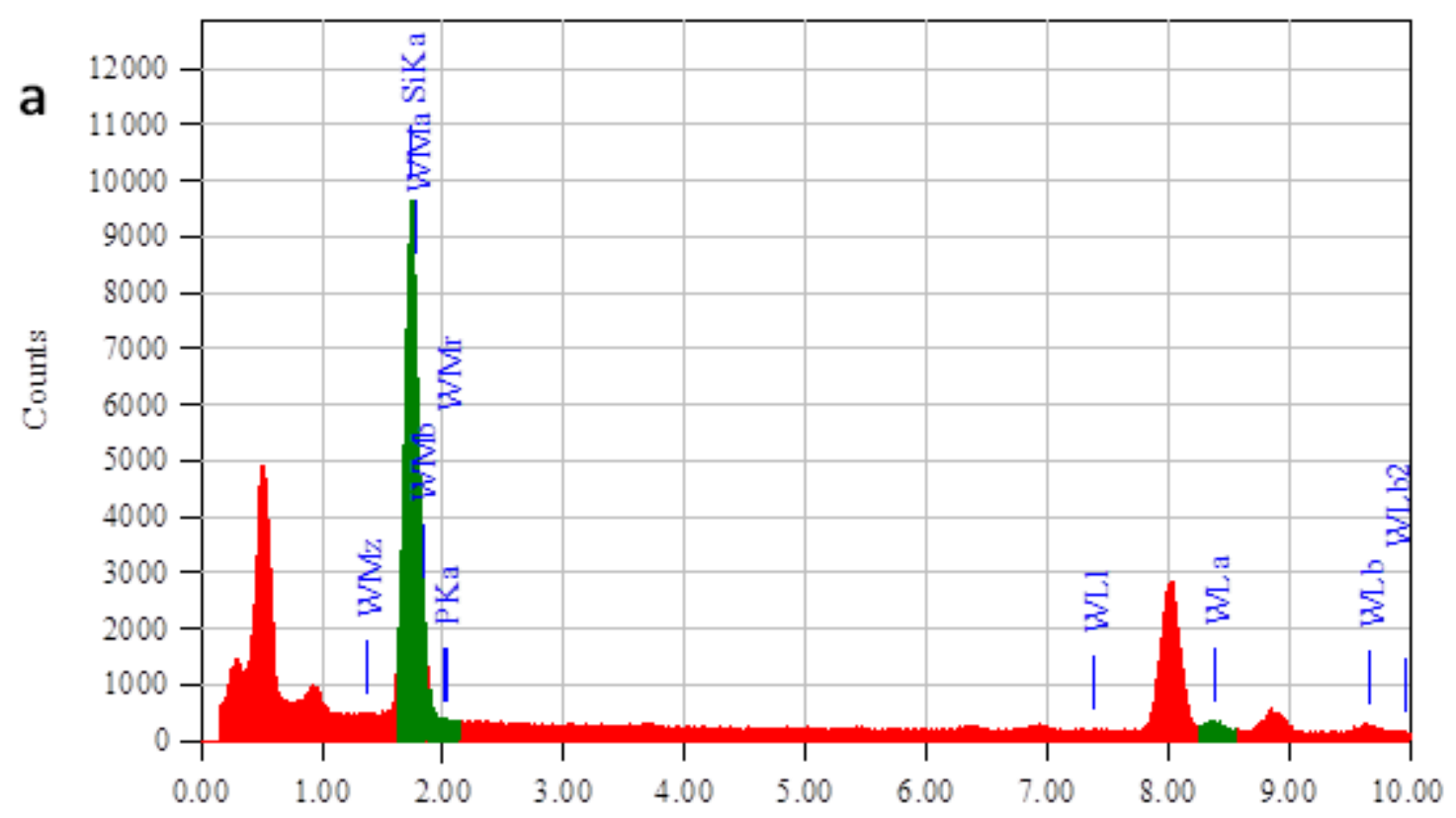

$\mathrm{keV}$

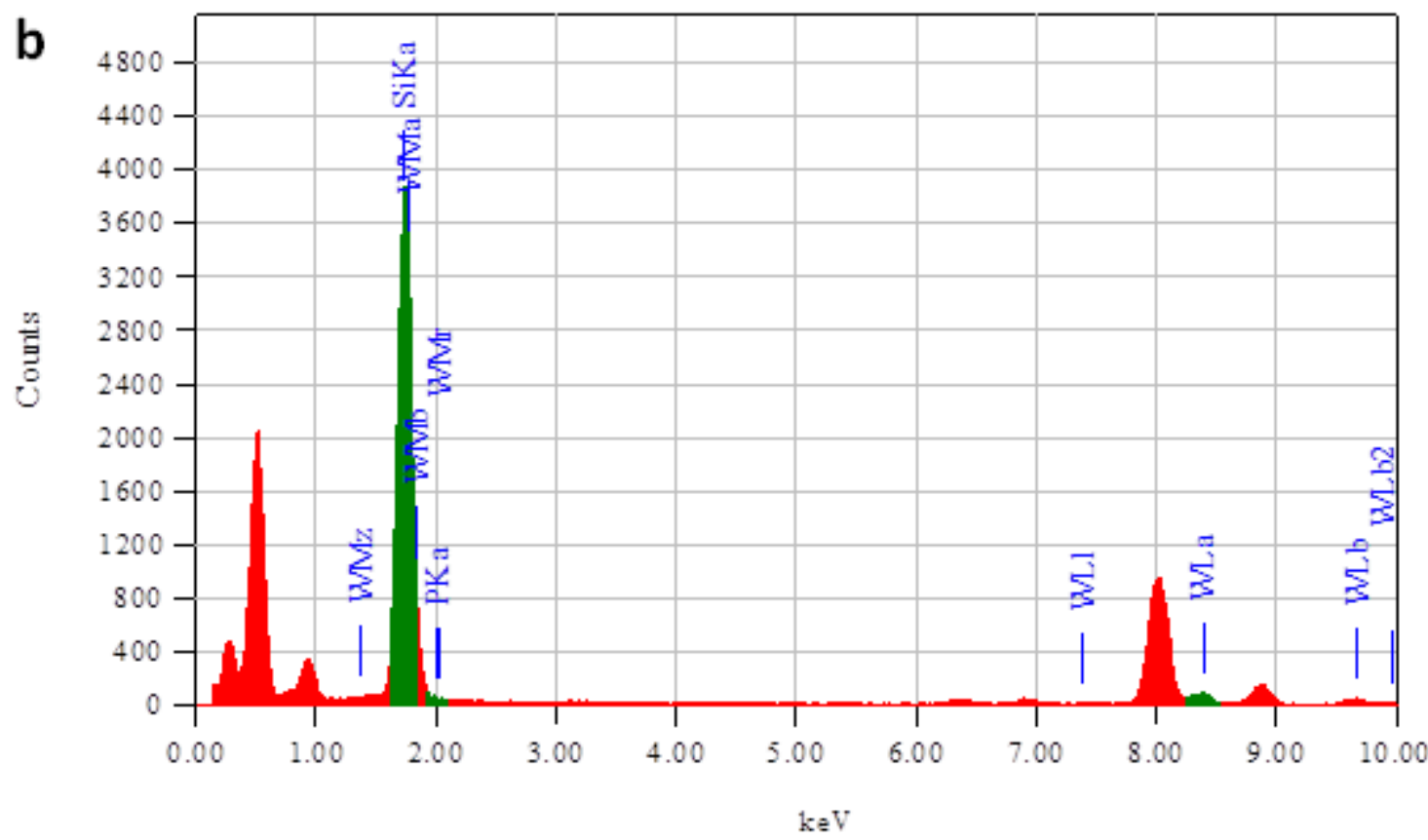

Fig.2.

EDX spectra of silica NANOPOM: a) twisted ribbons ( $L$ )-8 and b) helical ribbons $(L)-\mathbf{9}$. 
a)

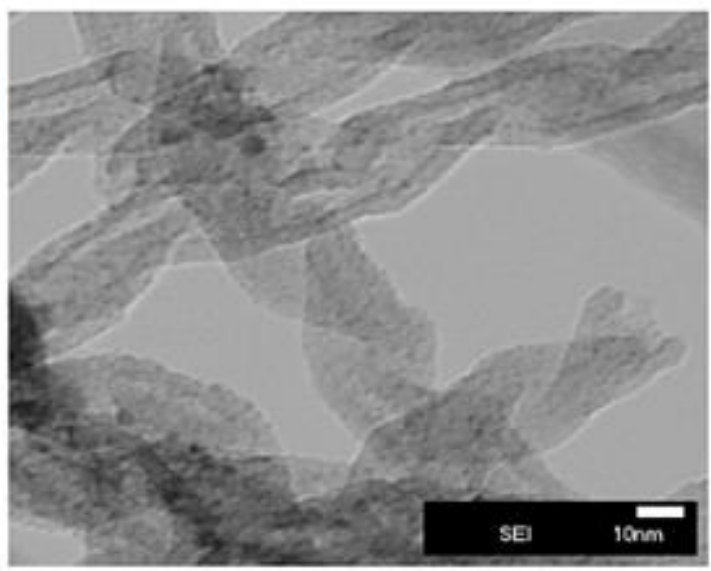

c)

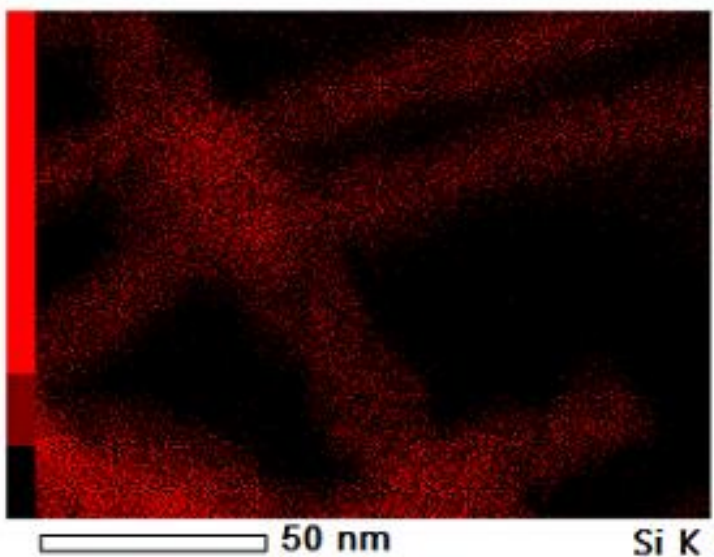

b)

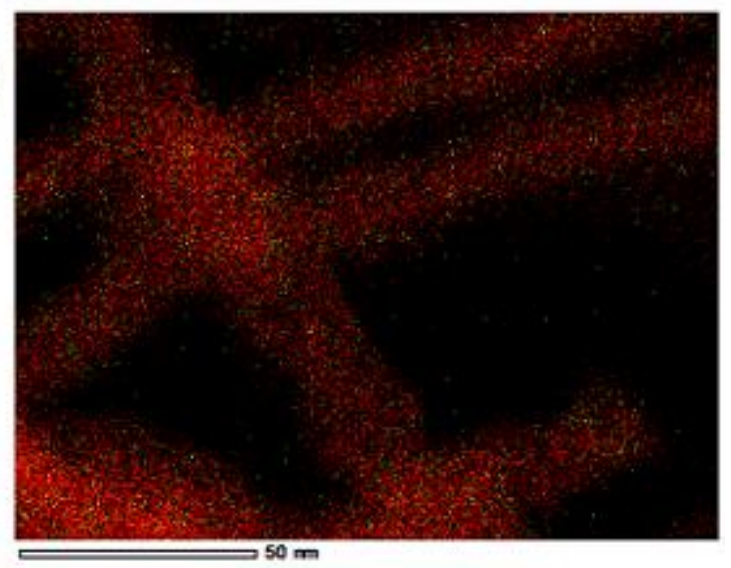

d)

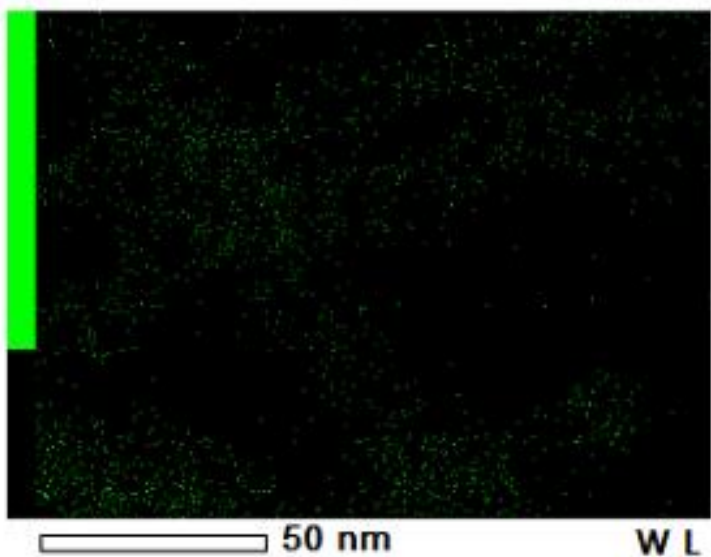

Fig.3.

a) STEM images of silica helical nanoribbons $(L)-9$; b) Elemental mappings of tungsten (W) and silicon ( $\mathrm{Si})$; c) Elemental mapping of $\mathrm{Si}$; d) Elemental mapping of $\mathrm{W}$. 


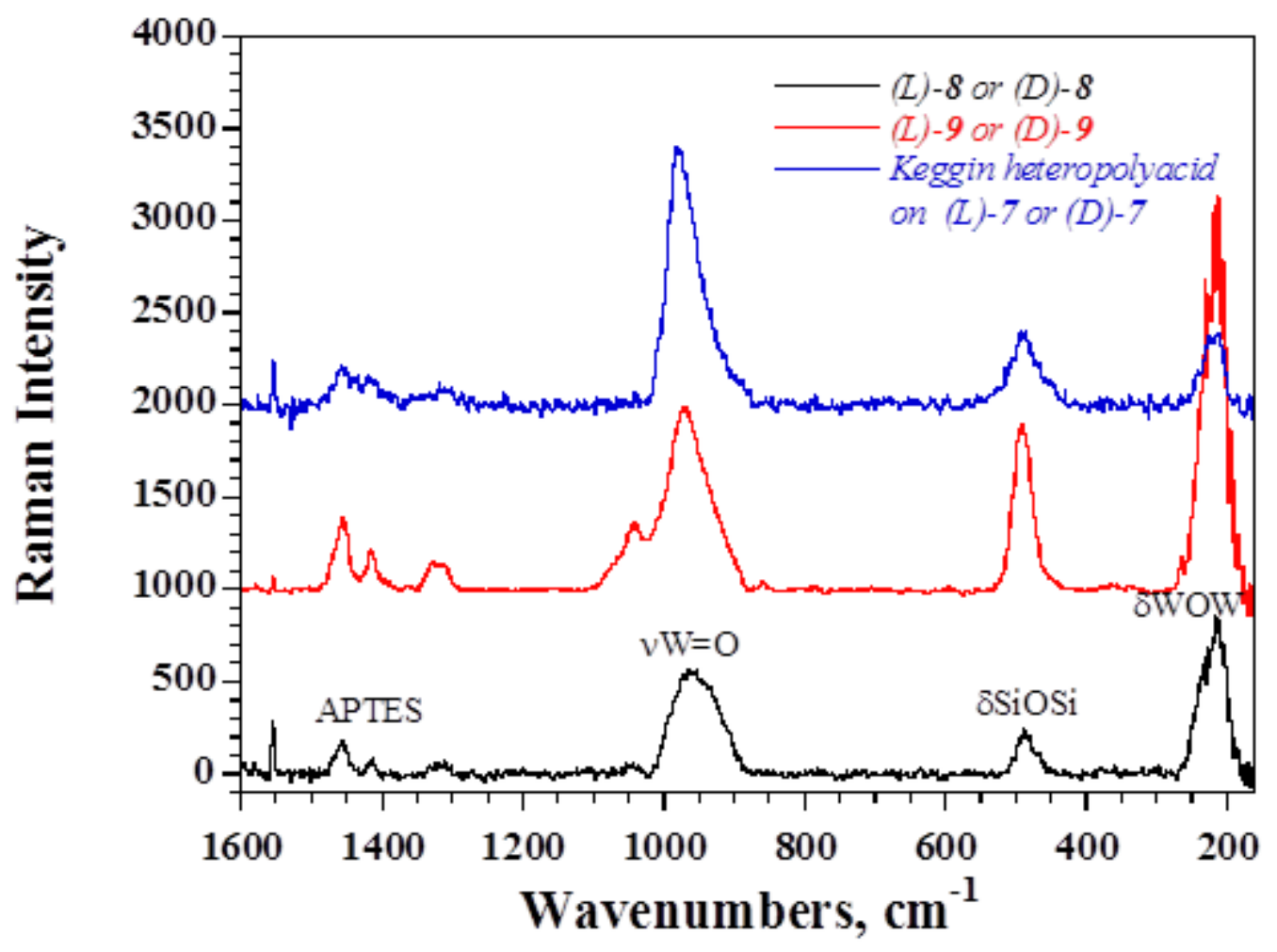

Fig.4.

Raman spectra of silica twisted ribbons $(L)$-8 (black spectrum), silica helical ribbons $(L)-\mathbf{9}$ (red spectrum), and Keggin heteropolyacid immobilized onto $(L)-7$ (blue spectrum) 
a)

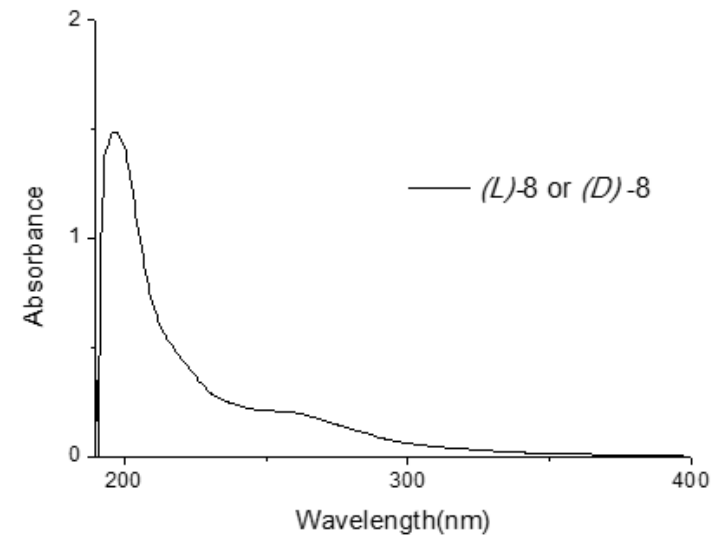

b)

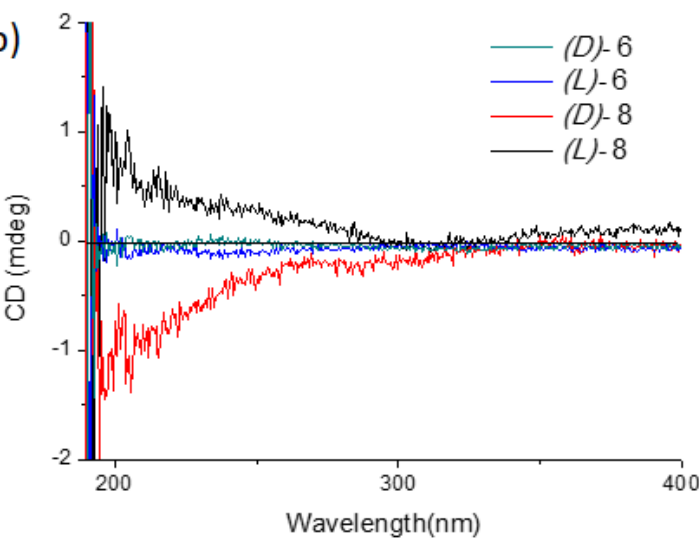

\section{Fig.5.}

a) UV-Vis spectra of silica NANOPOM twisted ribbons $(L)-8$ or $(D)-8$ in acetonitrile; b) CD spectra of APTES functionalized silica twisted ribbons before $(L)-\mathbf{6},(D)-\mathbf{6}$, and after mixing with POM $(L)-8,(D)-8$ in acetonitrile 


\section{Table 1}

Oxidation of methyl phenyl sulfide $\mathbf{1 0}$ catalyzed by silica NANOPOM hybrids $\mathbf{8}$ and $\mathbf{9}$, using $\mathrm{H}_{2} \mathrm{O}_{2}{ }^{a}$

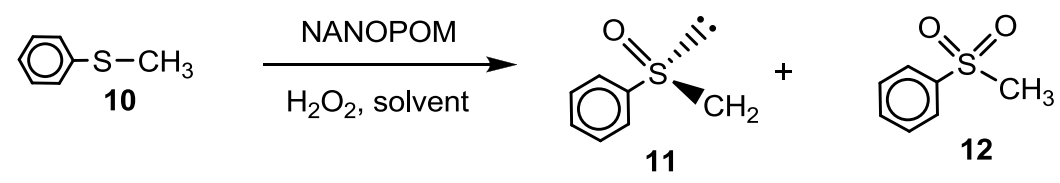

\begin{tabular}{|c|c|c|c|c|c|c|c|}
\hline \multirow[t]{2}{*}{ Entry } & \multirow[t]{2}{*}{ Catalysts (mol-\%) } & \multirow[t]{2}{*}{ solvent } & \multirow[t]{2}{*}{ Temperature $\left({ }^{\circ} \mathrm{C}\right)$} & \multirow[t]{2}{*}{ Time $(\min )^{\mathrm{b}}$} & \multirow{2}{*}{$\begin{array}{c}\text { Conversion } \\
(\%)^{\mathrm{c}}\end{array}$} & \multicolumn{2}{|c|}{ Products $(\%)$} \\
\hline & & & & & & $11^{\mathrm{d}}$ & 12 \\
\hline 1 & $L-(\mathbf{8})$ & $\mathrm{CH}_{3} \mathrm{CN}$ & -30 & 30 & 100 & 89 & 11 \\
\hline 2 & & & -30 & 60 & 100 & 66 & 34 \\
\hline 3 & & & 0 & 15 & 100 & 68 & 32 \\
\hline 4 & & Acetone & -30 & 15 & 26 & 26 & 0 \\
\hline 5 & & & -30 & 240 & 90 & 75 & 15 \\
\hline 6 & $L-(\mathbf{9})$ & $\mathrm{CH}_{3} \mathrm{CN}$ & 0 & 30 & 100 & 43 & 57 \\
\hline
\end{tabular}

${ }^{a}$ Reaction conditions: catalyst $(0.2 \mathrm{~mol} \%)$, substrate (250 equiv.), $\mathrm{H}_{2} \mathrm{O}_{2}(35 \%, 300$ eq), solvent ( $1 \mathrm{~mL})$.

${ }^{\mathrm{b}}$ Reactions were monitored by ${ }^{1} \mathrm{H}$ NMR.

${ }^{c}$ Conversion determined from the relative intensities of the ${ }^{1} \mathrm{H}$ NMR signals of the substrate and the product.

${ }^{\mathrm{d}}$ Racemic form 


\section{Graphical abstract}

Silica twisted and helical nanoribbons were prepared and used as chirality inducers for peroxophosphotungstate anions. Their morphologies, structural compositions and the presence of POM species were confirmed by UV-Vis, Raman, TEM, HR-TEM, and EDX characterization. Solid ${ }^{31}$ P NMR studies indicate the presence of the peroxophosphotungstate anion within within the structure of these NANOPOMs. Their optical activities were confirmed by circular dichroism (CD) spectroscopy, highlighting the chirality induction from chiral nanoribbons to achiral POM clusters, and their catalytic performances were evaluated in the heterogeneous oxidation of methyl phenyl sulfide as a model reaction, indicating NANOPOM built from twisted ribbons as the most efficient catalysts.

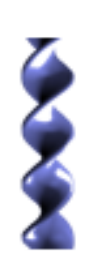

Silica Nanoribbon

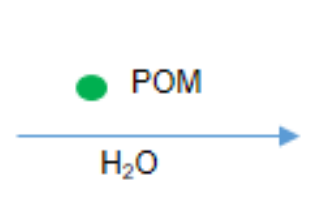

Silica NANOPOM

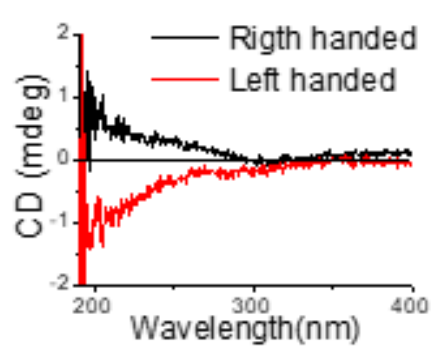




\section{Graphical abstract}

Silica twisted and helical nanoribbons were prepared and used as chirality inducers for peroxophosphotungstate anions. Their morphologies, structural compositions and the presence of POM species were confirmed by UV-Vis, Raman, TEM, HR-TEM, and EDX characterization. Solid ${ }^{31}$ P NMR studies indicate the presence of the peroxophosphotungstate anion within within the structure of these NANOPOMs. Their optical activities were confirmed by circular dichroism (CD) spectroscopy, highlighting the chirality induction from chiral nanoribbons to achiral POM clusters, and their catalytic performances were evaluated in the heterogeneous oxidation of methyl phenyl sulfide as a model reaction, indicating NANOPOM built from twisted ribbons as the most efficient catalysts.

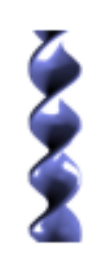

Silica Nanoribbon

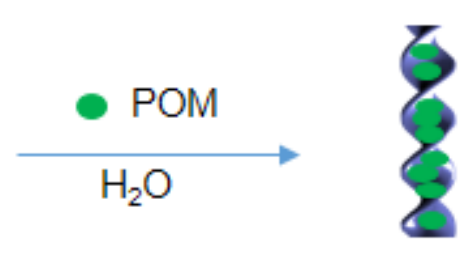

Silica NANOPOM

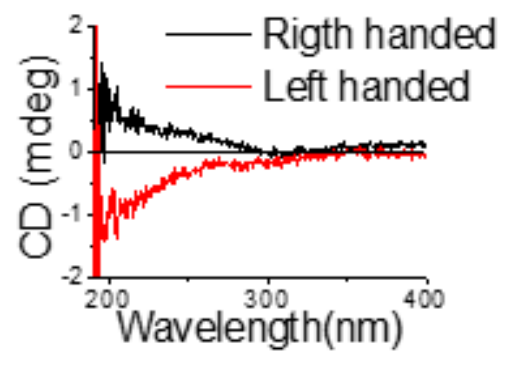

